\title{
STABILIZATION OF AN EPIDEMIC MODEL VIA AN $N$-PERIODIC APPROACH
}

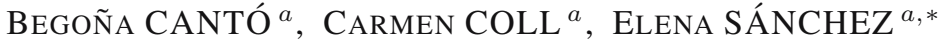 \\ ${ }^{a}$ Institute for Multidisciplinary Mathematics \\ Technical University of Valencia, Camino de Vera, 14, 46022 Valencia, Spain \\ e-mail: esanchezj@mat.upv.es
}

\begin{abstract}
We analyze the evolution of an infectious disease by combining different groups of a population when the route of transmission is via encounters with free-living virulent organisms that can survive for a long time outside the individual. This study involves matrix analysis of lower triangular block matrices and some of their spectral properties. We propose an $N$-periodic control strategy in order to stabilize the disease around the disease-free equilibrium point.
\end{abstract}

Keywords: nonnegative matrix, stability, reducible matrix, seasonality, epidemic process.

\section{Introduction}

In practice, the use of structured mathematical systems is common to model the propagation of an infectious disease through a population. To apply a model to real situations, we need to know several characteristics of the disease in question. In particular, this is the case when the dynamics of an infectious disease depend on the structure of a population, e.g., when the contagion of a susceptible individual occurs at a local level. The disease can be controlled using a variety of control strategies such as vaccination (Cantó et al., 2014; Ding et al., 2014; Liao and Yang, 2013).

In a typical herd the individuals of a species are organized in multiple groups (van den Driessche and Watmough, 2002; Xiao et al., 2006). For example, in a dairy herd, we have weanling calves, heifers and mature cows. Another example is a henhouse of laying hens; the birds are usually distributed into three areas: newly hatched chickens from 0 to 3 weeks, laying hens from 4 to 21 weeks, and sexually mature hens from 22 weeks. This distribution is necessary because suitable conditions of a ventilation system or an ambient temperature corresponding to the hen age must be guaranteed in the henhouse area. After a period of time, for instance, a week in the henhouse case, the individuals are changed to the next age-range cage. When a virulent organism is introduced in the farm, its population is subject to infection through contact with the pathogen that lives

* Corresponding author freely in the environment (Joh et al., 2009). This occurs, e.g., when an outbreak of Salmonella appears in a farm or in a henhouse (Prevost et al., 2006; Xiao et al., 2006) or when an outbreak of ovine brucellosis occurs in a herd (Ainseba et al., 2010).

In this paper, we study the behavior of a structured mathematical model incorporating the contamination by the environment as an indirect mode of transmission of the disease. We focus on the analysis of an outbreak of Salmonella at a henhouse, since this disease causes serious disorders in the population, as reported in the Annual Epidemiological Report 2014 on the evolution of Salmonella. In particular, in 2012, 92438 confirmed salmonellosis cases were reported by 29 EU/EEA countries. Through the analysis of how this disease has evolved, a decrease in reported cases can be observed. This is largely due to the implementation of policies for the control and prevention of salmonellosis in the poultry industry, particularly in laying hens and broilers (EFSA/ECLC, 2014).

In some cases, it is interesting to represent the epidemic process using a discrete-time model, since it allows us to take into account arbitrary time-step units and it is well linked to the real data collection discrete process (Cantó et al., 2013; 2014; Enatsu et al., 2012; Li and Wang, 2005). In the study of these discrete processes, matrix theory plays an important role, in particular the spectral properties of nonnegative matrices. This is because the variables involved in the process take nonnegative values. The concepts of reducible and 
irreducible matrices, a stable matrix, the Perron root, etc. (Berman and Plemmons, 1994) are needed to increase our knowledge of the evolution of the disease considered.

This paper is organized as follows. The next section contains mathematical and control preliminaries. In Section 3, we obtain theoretical results on the spectral radius of the structured matrices involved in the process. In Section 4, we present our compartmental epidemic model. In Section 5, we make the analysis of our model, the evolution of disease around the disease-free equilibrium point and the distribution of the population in multiple groups. We obtain some conditions which determine whether or not the disease is eradicated. Finally, in Section 6, we discuss the extinction of the disease by means of a seasonal strategy in the process, and the results are illustrated by an example.

\section{Preliminaries}

A matrix $A=\left(a_{i j}\right)$ is nonnegative (positive) if $a_{i j} \geq 0$ $\left(a_{i j}>0\right)$ for all $i, j$, and it is denoted by $A \geq O(A>O)$. The spectrum of $A$ and its spectral radius are denoted by $\sigma(A)$ and $\rho(A)$, respectively. It is known (Berman and Plemmons, 1994) that a matrix $A$ is stable if $\rho(A)<1$, and two matrices $A$ and $B$ are similar if there exists a nonsingular matrix $T$ such that $B=T^{-1} A T$. Moreover, $A$ is a reducible matrix if, for some permutation $T, A$ is similar to

$$
T^{T} A T=\left(\begin{array}{cc}
X & O \\
Y & Z
\end{array}\right),
$$

where $X$ and $Z$ are square matrices.

The following definitions and results constitute the foundations of stability theory of control systems (see, e.g., Meyer and Burrus, 1975). An autonomous discrete-time linear system $x(t+1)=A x(t)$ is asymptotically stable to zero if and only if $A$ is a stable matrix, that is, $\rho(A)<1$. In the $N$-periodic case, $N \in \mathbb{Z}$, the system is $x(t+1)=A(t) x(t)$, with $A(t+N)=$ $A(t), t \in \mathbb{Z}$. In this case stability can be assessed by analyzing the monodromy matrix defined as the transition matrix over one period, i.e.,

$$
\Phi_{A}\left(k, k_{0}\right)= \begin{cases}\prod_{\substack{i=k_{0} \\ J_{n},}}^{k-1} A\left(k_{0}+k-1-i\right), & k>k_{0}, \\ & k=k_{0},\end{cases}
$$

where $J_{n}$ denotes the identity matrix of size $n$. Thus, the periodic system is asymptotically stable to zero if and only if the $N$ matrices $A_{s}=\Phi_{A}(N+s, s), s=0,1, \ldots, N-1$ are stable. Moreover, Bittanti (1986) proved that these matrices have the same spectrum.

The problem presented in this paper refers to the evolution of a disease in an epidemic SIC model described by a discrete system involving matrices with a fixed structure. The behavior of the disease will be analyzed through the evolution of the infected individual and contaminant populations. We are interested in establishing procedures or strategies that modify these variables and in setting up some actions to ensure that the disease tends to disappear. The particular structure of the matrices associated with the infected individuals and the contaminant populations in the linear model leads us to deepening the study of the spectral properties of such matrices. In the next section we are concerned with the properties of a nonnegative matrix with lower triangular blocks. Therefore, before approaching the study of this epidemiological model, we develop some of these properties.

\section{Nonnegative matrices with lower triangular blocks}

Consider a reducible nonnegative matrix $E=\left(e_{i j}\right) \in$ $\mathbb{R}_{+}^{m \times m}$. This statement is not restrictive since the existence of a reducible infection matrix is common in epidemic models (Rass and Radcliffe, 2000). It is known (Berman and Plemmons, 1994) that there exists a permutation matrix such that $E$ is similar to its Frobenius normal form which is a lower triangular block matrix whose square blocks of the diagonal are either irreducible or a $1 \times 1$ null matrix. In this work we focus our attention on reducible matrices $E \in \mathbb{R}_{+}^{2 n \times 2 n}$ such that their Frobenius normal form is given by

$$
\tilde{E}=\left(\begin{array}{cccc}
\tilde{E}_{1} & O & O & O \\
\star & \tilde{E}_{2} & O & O \\
\vdots & \vdots & \ddots & \vdots \\
\star & \star & \cdots & \tilde{E}_{n}
\end{array}\right)
$$

where $\tilde{E}_{i} \in \mathbb{R}_{+}^{2 \times 2}$ are irreducible matrices, $i \in \Upsilon$, with $\Upsilon=\{1,2, \ldots, n\}$, and where the stars represent suitable block matrices obtained to construct $E$.

We denote by $\mathcal{E}$ the following set:

$$
\begin{aligned}
\mathcal{E}=\{ & E=\left(\begin{array}{cc}
E_{1} & E_{2} \\
E_{3} & E_{4}
\end{array}\right) \mid E_{i} \in \mathbb{R}_{+}^{n \times n} \text { lower } \\
& \text { triangular with positive diagonal entries }\} .
\end{aligned}
$$

In the following result we establish that the nonnegative matrices $E \in \mathbb{R}_{+}^{2 n \times 2 n} \in \mathcal{E}$ have the Frobenius normal form given in (1).

Proposition 1. The nonnegative matrix $E \in \mathcal{E}$ is reducible and its Frobenius normal form is given by (1).

Proof. If we construct the permutation matrix

$$
U=\left(\begin{array}{llllllll}
u_{1} & u_{3} & \ldots & u_{2 n-1} & u_{2} & u_{4} & \ldots & u_{2 n}
\end{array}\right),
$$

$u_{i}$ being the $i$-th unit vector, it is easy to check that $\tilde{E}=$ $U E U^{T}$ is a lower triangular block matrix. The matrix $U$ 
leads to the following reordering of the entries of matrix $E=\left(e_{i j}\right)$ :

$$
\begin{array}{rlrl}
e_{j j} & =\tilde{e}_{2 j-1,2 j-1}, & e_{n+j, n+j} & =\tilde{e}_{2 j, 2 j}, \\
e_{n+j, j} & =\tilde{e}_{2 j, 2 j-1}, \quad e_{j, n+j} & =\tilde{e}_{2 j-1,2 j}, \quad j \in \Upsilon .
\end{array}
$$

Then, under the assumption that matrices $E_{i}$ have positive diagonal entries, all blocks $\tilde{E}_{i}$ are positive matrices, and thus irreducible ones. Hence, the matrix $E$ is a reducible matrix and the matrix $\tilde{E}$ given in 10 is its Frobenius normal form.

We denote by $\mathcal{X}$ the set of matrices with a real and nonnegative spectrum defined by

$$
\begin{aligned}
\mathcal{X}=\{ & X=\left(x_{i j}>0\right) \in \mathbb{R}_{+}^{2 \times 2}, \\
& \text { with } \left.\sigma(X)=\left\{\lambda_{1}, \lambda_{2}\right\}, 0 \leq \lambda_{2}<\lambda_{1}\right\} .
\end{aligned}
$$

\section{Proposition 2.}

1. Consider $X \in \mathcal{X}$. If $x_{11}+x_{22}<1$, then $\rho(X)<1$.

2. Consider a nonnegative matrix $E=\left(e_{i j}\right) \in \mathcal{E}$ such that $e_{i i}+e_{n+i, n+i}<1$ and $e_{i, n+i} e_{n+i, i} \leq$ $e_{i i} e_{n+i, n+i}$, for all $i \in \Upsilon$; then $\rho(E)<1$.

Proof.

Part 1. If the spectral radius of $X, \lambda_{1}=\rho(X)$, is greater than 1 , using algebraic calculations we have

$$
\begin{aligned}
& \lambda_{1}= \rho(X)=\frac{1}{2}\left(x_{11}+x_{22}\right. \\
&\left.+\sqrt{\left(x_{11}-x_{22}\right)^{2}+4 x_{12} x_{21}}\right) \geq 1, \\
&\left(x_{11}-x_{22}\right)^{2}+4 x_{12} x_{21} \geq\left(2-x_{11}-x_{22}\right)^{2}, \\
& \\
& 1-\left(x_{11}+x_{22}\right)+\operatorname{det}(X) \leq 0 .
\end{aligned}
$$

However, this contradicts the hypothesis, since $\operatorname{det}(X)=\lambda_{1} \lambda_{2} \geq 0$ because $X \in \mathcal{X}$ and, by the hypothesis, $x_{11}+x_{22}<1$.

Part 2. By Proposition 1 each diagonal block $\tilde{E}_{i}=\left(\tilde{e}_{k l}^{i}\right)$ of $\tilde{E}=U E U^{T}$ has a nonnegative spectrum, that is, $\tilde{E}_{i} \in$ $\mathcal{X}$, and satisfies $\tilde{e}_{11}^{i}+\tilde{e}_{22}^{i}<1$; then $\rho\left(\tilde{E}_{i}\right)<1$, for all $i \in \Upsilon$. Hence, $\rho(E)<1$.

Now, we consider the matrix

$$
Q_{n}=\left(\begin{array}{cc}
J_{n} & O \\
O & O
\end{array}\right) \in \mathbb{R}^{2 n \times 2 n}
$$

for all $n \geq 1$. We want to analyze spectral properties of the product of matrices $E^{N} Q_{n}, N \in \mathbb{N}$. Specifically, we look for the greatest value of $N$ such that $\rho\left(E^{N} Q\right)<1$ and $\rho\left(E^{N+1} Q_{n}\right)>1$, as $E^{N} Q_{n}=U^{T} \tilde{E}^{N} \operatorname{diag}\left(Q_{1}\right) U$, and

$$
\tilde{E}^{N} \operatorname{diag}\left(Q_{1}\right)=\left(\begin{array}{cccc}
\tilde{E}_{1}^{N} Q_{1} & O & O & O \\
\star & \tilde{E}_{2}^{N} Q_{1} & O & O \\
\vdots & \vdots & \ddots & \vdots \\
\star & \star & \cdots & \tilde{E}_{n}^{N} Q_{1}
\end{array}\right),
$$

where the stars represent suitable block matrices obtained to construct $\tilde{E}^{N} \operatorname{diag}\left(Q_{1}\right)$. We focus our attention on the blocks $\tilde{E}_{i}^{N} Q_{1}$.

Proposition 3. Consider $X \in \mathcal{X}$ with $\rho(X) \geq 1$. If $x_{11}<1$, then there exists $N_{0} \in \mathbb{N}$ such that $\rho\left(X^{N} Q_{1}\right)<$ 1 for all $N \leq N_{0}$ and $\rho\left(X^{N} Q_{1}\right)>1$ when $N>N_{0}$.

Proof. It is straightforward that the nonzero eigenvalue of $X^{N} Q_{1}$ is given by

$$
\sigma_{N}=\frac{\lambda_{1}^{N}\left(\lambda_{1}-x_{22}\right)+\left(x_{22}-\lambda_{2}\right) \lambda_{2}^{N}}{\lambda_{1}-\lambda_{2}}
$$

and $\rho\left(X^{N} Q_{1}\right)=\sigma_{N}$, for all $N \in \mathbb{N}$. This is obtained using the eigenvectors

$$
v_{i}=\left(\frac{\lambda_{i}-x_{22}}{x_{21}}, 1\right), \quad i=1,2
$$

associated with $\lambda_{1}$ and $\lambda_{2}$, respectively. Since $0<\lambda_{2}<$ $\lambda_{1}$, we have $\sigma_{N}=\rho\left(X^{N} Q_{1}\right)>0$.

Consider $\rho(X)=\lambda_{1}=1$. As $\lambda_{2}<\lambda_{1}=1$, we have $\lambda_{2}^{N}<1$. Hence

$$
\left(1-x_{22}\right)+\left(x_{22}-\lambda_{2}\right) \lambda_{2}^{N}<1-\lambda_{2} .
$$

Using (2), we obtain $\sigma_{N}<1$.

Now, we consider $\rho(X)=\lambda_{1}>1$ and construct the following continuous function:

$$
f(z)=1-a\left(1-\frac{1}{\lambda_{1}^{z}}\right)-\left(\frac{\lambda_{2}}{\lambda_{1}}\right)^{z}
$$

with

$$
a=\frac{\lambda_{1}-\lambda_{2}}{x_{22}-\lambda_{2}},
$$

$z \in \mathbb{R}_{+}$, whose $f^{\prime}(z)$ has a unique root. From the definition of the set $\mathcal{X}$ we have $\lambda_{1}-\lambda_{2}>0$. By performing a simple calculation it is verified that

$$
\begin{aligned}
& x_{22}-\lambda_{2} \\
& =x_{22}-\frac{1}{2}\left(x_{11}+x_{22}-\sqrt{\left(x_{11}-x_{22}\right)^{2}+4 x_{12} x_{21}}\right) \\
& =\frac{1}{2}\left(\sqrt{\left(x_{11}-x_{22}\right)^{2}+4 x_{12} x_{21}}-\left(x_{11}-x_{22}\right)\right)>0,
\end{aligned}
$$

since $x_{12}, x_{21}>0$. Moreover, by the hypothesis, $1+$ $x_{22}-\lambda_{1}-\lambda_{2}=1-x_{11}>0$. Then the function (3) is 
positive at $z=1$, that is,

$$
\begin{aligned}
f(1) & =1-a\left(1-\frac{1}{\lambda_{1}}\right)-\frac{\lambda_{2}}{\lambda_{1}} \\
& =\frac{\left(\lambda_{1}-\lambda_{2}\right)\left(1+x_{22}-\lambda_{1}-\lambda_{2}\right)}{\left(x_{22}-\lambda_{2}\right) \lambda_{1}}>0 .
\end{aligned}
$$

On the other hand, this fact, along with with the assumption $\lambda_{2} \geq 0$, guarantees that

$$
1-a\left(1-\frac{1}{\lambda_{1}}\right)>0
$$

Hence

$$
\lambda_{1}<\frac{a}{a-1} .
$$

Consequently, there exists $z_{1}>1, z_{1} \in \mathbb{R}_{+}$such that

$$
\lambda_{1}^{z}>\frac{a}{a-1}
$$

for all $z>z_{1}$. In this case, from the construction of $f(z)$, we also have $f(z)<0$ if $z>z_{1}$. Thus, there exists a unique $z_{0} \in \mathbb{R}_{+}, 1<z_{0}<z_{1}$ such that $f\left(z_{0}\right)=0$.

Taking $N_{0}=\operatorname{int}\left(z_{0}\right)$, where 'int' denotes the integer part of $z_{0}$, we have $f(N)>0$ if $N \leq N_{0}$, and otherwise $f(N)<0$.

From (3), if $N \leq N_{0}$, we get

$$
\begin{aligned}
a & >\lambda_{1}^{N}\left(\left(\frac{\lambda_{2}}{\lambda_{1}}\right)^{N}+a-1\right), \\
\lambda_{1}-\lambda_{2} & >\lambda_{2}^{N}\left(x_{22}-\lambda_{2}\right)+\lambda_{1}^{N}\left(\lambda_{1}-x_{22}\right), \\
1 & >\sigma_{N} .
\end{aligned}
$$

Thus, $\rho\left(X^{N} Q_{1}\right)<1$ for all $N \leq N_{0}$. Likewise, if $N>$ $N_{0}$, we obtain $\rho\left(X^{N} Q_{1}\right)>1$.

Theorem 1. Let $E \in \mathbb{R}_{+}^{2 n \times 2 n}$ be a reducible matrix with its Frobenius normal form given in (1) and $\tilde{E}_{i}=$ $\left(\tilde{e}_{k l}^{i}\right) \in \mathcal{X}$ with $\tilde{e}_{11}^{i}<1$. If $\rho(E) \geq 1$, then there exists $N_{0} \in \mathbb{N}$ such that $\rho\left(E^{N} Q_{n}\right)<1$ for all $N \leq N_{0}$ and $\rho\left(E^{N} Q_{n}\right)>1$ when $N>N_{0}$.

Proof. Since $\rho(E) \geq 1$, there exists some $i \in \Upsilon$ such that $\rho\left(\tilde{E}_{i}\right)=\lambda_{1}^{i} \geq 1$. Let $\Upsilon_{1}=\left\{i \in \Upsilon \mid \lambda_{1}^{i} \geq 1\right\}$. For each $i \in \Upsilon_{1}$, applying Proposition 3 to the matrix $\tilde{E}_{i}$, there exists $N_{0}^{i}$ such that $\rho\left(\tilde{E}_{i}^{N} Q_{1}\right)<1$ for all $N \leq N_{0}^{i}$ and $\rho\left(\tilde{E}_{i}^{N} Q_{1}\right)>1$ when $N>N_{0}^{i}$. Thus, it is sufficient to take $N_{0}=\min \left\{N_{0}^{i}, i \in \Upsilon_{1}\right\}$.

Note that in the previous results we obtained the existence and uniqueness of $N_{0}$. In practice, we need to apply numerical methods to estimate the values $N_{0}^{i}$, $i \in \Upsilon_{1}$. In what follows we give a procedure to determine the minimum power $N_{0}$ that is recommended to be carried out from an initial unstable matrix $E$ into a stable $E_{0}^{N} Q_{n}$ matrix.
Algorithm 1. Minimum period to maintain stability.

Step 1. Input data: $E$ (reducible matrix, $E \in$ $\mathcal{E}$ ), $n$ (size of $E$ ) and $U$ (permutation matrix, $U=$ $\left.\left(u_{1} u_{3} \ldots u_{2 n-1} u_{2} u_{4} \ldots u_{2 n}\right)\right)$.

Step 2. Compute $\rho(E)$ (spectral radius of $E$ ).

Step 2.1. If $\rho(E)<1$ then go to Step 8 .

Step 2.2. Otherwise, go to Step 3 .

Step 3. Construct $\tilde{E}=U E U^{T}$.

Step 4. Extract the blocks $\tilde{E}_{i}=\left(\tilde{e}_{k l}^{i}\right)$ of $\tilde{E}, i \in \Upsilon$.

Step 5. Set $i=1$ :

Step 5.1. Compute $\lambda_{1}^{i}=\rho\left(\tilde{E}_{i}\right), \lambda_{2}^{i}$ as the eigenvalues of $\tilde{E}_{i}$.

Step 5.2. If $\lambda_{1}^{i} \leq 1$, then $N_{0}^{i}=1$ and go to Step 6 , otherwise, go to Step 5.3.

Step 5.3. Compute $\tilde{e}_{22}^{i}$ and set

$$
a=\frac{\lambda_{1}^{i}-\lambda_{2}^{i}}{\tilde{e}_{22}^{i}-\lambda_{2}^{i}} .
$$

Step 5.4. Construct

$$
f_{i}(z)=1-a\left(1-\frac{1}{\left(\lambda_{1}^{i}\right)^{z}}\right)-\left(\frac{\lambda_{2}^{i}}{\lambda_{1}^{i}}\right)^{z} .
$$

Step 5.5. Solve $f_{i}(z)=0$ and take the integer part of the solution. Store it in $N_{0}^{i}$ and go to Step 6.

Step 6. Set $i=i+1$. If $i \leq n$ go to Step 5.1. Otherwise, go to Step 7.

Step 7. Construct $N_{0}=\min \left\{N_{0}^{i}, i \in \Upsilon\right\}$.

\section{Problem statement}

We consider an epidemiological process where the individuals are organized in $n$ compartments depending on their age. In this model the individuals are infected only from the contact with the contaminant of its compartment. We denote by $P$ the population, by $S_{i}$ susceptible individuals, by $I_{i}$ infected individuals and by $C_{i}$ the contaminant population in the $i$-th compartment, for $i \in \Upsilon$.

We take into account transfer of individuals from the $i$-th compartment to the $(i+1)$-th compartment when they change the age range in the dynamic process and, moreover, we consider the entry of new individuals in $S_{1}$ proportional to the size of the population $\nu(t) P$, for a fixed size of the population $P$. This assumption is realistic since we are considering the poultry industry. To optimize resources and facilities, such as the automatic drinking or dispensing grain system and light management, the farmer is interested in having the maximum number of hens in the henhouse fit. 
A common mathematical representation for a nonlinear discrete-time system is

$$
x(t+1)=f(x(t)), \quad t \geq 0,
$$

where $x(t) \in \mathbb{R}^{n}$ is the state vector and $f: \mathbb{R}^{n} \rightarrow \mathbb{R}^{n}$ is a continuously differentiable function.

In our system, $x(t)=(S(t) I(t) C(t))^{T}$ with $S(t)=$ $\left(S_{i}(t)\right)_{i=1}^{n}, I(t)=\left(I_{i}(t)\right)_{i=1}^{n}, C(t)=\left(C_{i}(t)\right)_{i=1}^{n}$. The parameters involved in the model are the following:

- $p_{i}, q_{i}, s_{i}$ : survival rates of $S_{i}, I_{i}, C_{i}$, respectively;

- $\alpha_{i}$ : exposition rate of susceptible individuals $S_{i}$ by contact with $C_{i}$;

- $\sigma_{i}\left(\mu_{i}\right)$ : rate at which individuals $S_{i}\left(I_{i}\right)$ change the age-compartment without changing the state, i.e., those who remain susceptible individuals (infected individuals);

- $\epsilon_{i}$ : rate at which the individuals change the age-compartment, with a change from susceptible to infected;

- $\beta_{i}$ : rate at which of the contaminant is produced by infected individuals $I_{i}$.

Having described the parameters involved in the model, we proceed to discuss in detail its construction. In the model, susceptible individuals $S_{i}$ of the $i$-th compartment have three options when their situation changes: they can advance to the next $S_{i+1}$ of susceptible individuals (if they have not become infected but have changed their age), they can move to infected individuals $I_{i}$ (if they have not changed age but they have become infected) or they can advance to the infected individuals $I_{i+1}$ of the $(i+1)$-th compartment (they just change their age when they become infected). The number of the individuals of each option is given by $\sigma_{i} S_{i}(t),(1-$ $\left.\epsilon_{i}\right) \alpha_{i} C_{i}(t) S_{i}(t)$ and $\epsilon_{i} \alpha_{i} C_{i}(t) S_{i}(t)$, respectively. This reasoning is summarized in the scheme given in Fig. 1

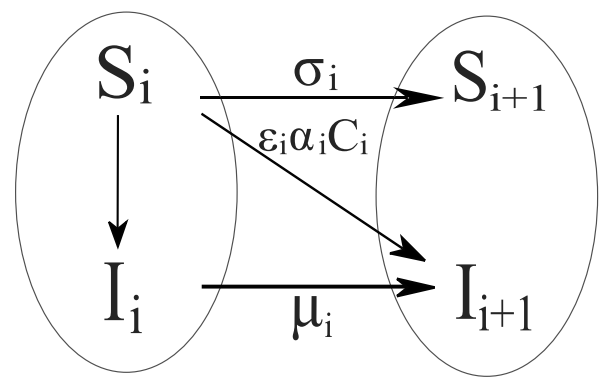

Fig. 1. Distribution scheme.

Specifically, the nonlinear discrete-time system is given by

$$
\begin{aligned}
& \left\{\begin{aligned}
S_{1}(t+1)= & \left(p_{1}-\sigma_{1}-\alpha_{1} C_{1}(t)\right) S_{1}(t)+\nu(t) P, \\
S_{j}(t+1)= & \sigma_{j-1} S_{j-1}(t) \\
& +\left(p_{j}-\sigma_{j}-\alpha_{j} C_{j}(t)\right) S_{j}(t), \\
S_{n}(t+1)= & \sigma_{n-1} S_{n-1}(t)+\left(p_{n}-\alpha_{n} C_{n}(t)\right) S_{n}(t),
\end{aligned}\right. \\
& \left\{\begin{aligned}
I_{1}(t+1)= & \left(q_{1}-\mu_{1}\right) I_{1}(k) \\
& +\left(1-\epsilon_{1}\right) \alpha_{1} C_{1}(t) S_{1}(t), \\
I_{j}(t+1)= & \mu_{j-1} I_{j-1}(t)+\left(q_{j}-\mu_{j}\right) I_{j}(t) \\
& +\epsilon_{j-1} \alpha_{j-1} C_{j-1}(t) S_{j-1}(t) \\
& +\left(1-\epsilon_{j}\right) \alpha_{j} C_{j}(t) S_{j}(t), \\
I_{n}(t+1)= & \mu_{n-1} I_{n-1}(t)+q_{n} I_{n}(t) \\
& \epsilon_{n-1} \alpha_{n-1} C_{n-1}(t) S_{n-1}(t) \\
& +\alpha_{n} C_{n}(t) S_{n}(t), \\
C_{1}(t+1)= & s_{1} C_{1}(t)+\beta_{1}\left(1-\mu_{1}\right) I_{1}(t), \\
C_{j}(t+1)= & s_{j} C_{j}(t)+\mu_{j-1} \beta_{j-1} I_{j-1}(t) \\
& +\left(1-\mu_{j}\right) \beta_{j} I_{j}(t), \\
C_{n}(t+1)= & s_{n} C_{n}(t)+\mu_{n-1} \beta_{n-1} I_{n-1}(t) \\
& +\beta_{n} I_{n}(t),
\end{aligned}\right. \\
& \text { for } j=2, \ldots, n-1 \text {. }
\end{aligned}
$$

This system will be called the SIC model. Since the population is constant at any time, we have

$$
\begin{aligned}
\sum_{i=1}^{n} S_{i}(t)+\sum_{i=1}^{n} & I_{i}(t) \\
& =\sum_{i=1}^{n} S_{i}(t+1)+\sum_{i=1}^{n} I_{i}(t+1)=P .
\end{aligned}
$$

From (4), adding the corresponding equations, we have that the function $\nu(t)$ must meet

$$
\nu(t) P=P-\sum_{i=1}^{n} p_{i} S_{i}(t)-\sum_{i=1}^{n} q_{i} I_{i}(t) .
$$

Note that this equation determines the number of hens dying at each stage, i.e., the number of hens that the farmer must replace in every step.

This model seeks to analyze the behavior of infection through the evolution of infected individuals and the amount of the contaminant in the compartments. Our goal is to find and apply strategies via an $N$-periodic approach to ensure that the disease tends to disappear.

\section{Evolution of the disease in the SIC model}

Consider the model given in (4) and abbreviated as $x(t+$ $1)=f(x(t))$, where $x(t)=(S(t) I(t) C(t))^{T}$ with $S(t)=\left(S_{i}(t)\right)_{i=1}^{n}, I(t)=\left(I_{i}(t)\right)_{i=1}^{n}$ and $C(t)=$ $\left(C_{i}(t)\right)_{i=1}^{n}$. The equilibrium points $x^{\star}=\left(S^{*}, I^{*}, C^{*}\right)$ are the solutions of $x^{\star}=f\left(x^{\star}\right)$ and, specifically, when $I^{\star}=$ 0 , we have the disease-free equilibrium point obtained in the following proposition. 
Proposition 4. The disease-free equilibrium point of the model (4), $\left(S_{f}, O, O\right)$ with $S_{f}=\left(S_{1}^{f}, S_{2}^{f}, \ldots, S_{n}^{f}\right)$, provides the following distribution of the population:

$$
S_{i}^{f}=\frac{H_{i+1, n} \Sigma_{i} P}{H},
$$

where

$$
\begin{aligned}
\Sigma_{i}= & \prod_{j=1}^{i-1} \sigma_{j}, \quad i \in \Upsilon \\
H= & \left(1-p_{n}\right)\left(\left(1+\sigma_{1}\right) H_{2, n-1}\right. \\
& \left.+\sum_{j=2}^{n-1} p_{j} \Sigma_{j-1} H_{j+1, n-1}\right)+p_{n} \sigma_{n-1},
\end{aligned}
$$

and

$$
H_{k, l}=\prod_{j=k}^{l}\left(1-p_{j}+\sigma_{j}\right)
$$

if $k<l$, and $H_{k, l}$ is the identity matrix if $k>l$.

Proof. Now, we give a sketch of the proof. The system (4) at the disease-free equilibrium point $\left(S_{f}, O, O\right)$ is given by

$$
S\left\{\begin{array}{c}
S_{1}^{f}=\left(p_{1}-\sigma_{1}\right) S_{1}^{f}+P-\sum_{i=1}^{n} p_{i} S_{i}^{f}, \\
S_{j}^{f}=\sigma_{j-1} S_{j-1}^{f}+\left(p_{j}-\sigma_{j}\right) S_{j}^{f}, \\
j=2, \ldots, n-1, \\
S_{n}^{f}=\sigma_{n-1} S_{n-1}^{f}+p_{n} S_{n}^{f} .
\end{array}\right.
$$

Solving this system algebraically, we have

$$
\begin{aligned}
S_{1}^{f}= & \left(1-p_{n}\right)\left(1-p_{n-1}+\sigma_{n-1}\right) \\
& \cdots\left(1-p_{2}+\sigma_{2}\right) \frac{P}{L}, \\
S_{j}^{f}= & \left(1-p_{n}\right)\left(1-p_{n-1}+\sigma_{n-1}\right) \\
& \cdots\left(1-p_{i-1}+\sigma_{i-1}\right) \frac{P}{L} \sigma_{1} \sigma_{2} \cdots \sigma_{i-1}, \\
S_{n}^{f}= & \frac{P}{L} \sigma_{1} \sigma_{2} \cdots \sigma_{n-1},
\end{aligned}
$$

with

$$
\begin{aligned}
L= & \left(1+\sigma_{1}\right)\left(1-p_{n}\right)\left(1-p_{n-1}+\sigma_{n-1}\right) \\
& \cdots\left(1-p_{1}+\sigma_{1}\right)+p_{2} \sigma_{1} \\
& \left(1-p_{n}\right)\left(1-p_{n-2}+\sigma_{n-2}\right) \cdots\left(1-p_{1}+\sigma_{1}\right) \\
& +\cdots+p_{n} \prod_{i=1}^{n-l} \sigma_{i} \prod_{i=1}^{n}\left(1-p_{i}+\sigma_{i}\right) .
\end{aligned}
$$

Rearranging the terms, we get the solution (5).

Linearizing around the disease-free equilibrium point $x^{\star}=f\left(x^{\star}\right)$, with $P_{f}=\left(S^{f}, O, O\right) \in \mathbb{R}^{3 n}$, $S^{f}=\left(S_{1}^{f}, \ldots, S_{n}^{f}\right)$, we obtain the following linear discrete-time system:

$$
\hat{x}(t+1)=A \hat{x}(t)+b,
$$

with $\hat{x}(t)=x(t)-x^{\star}, b=\left(P O_{3 n-1}\right)^{T} \in \mathbb{R}^{3 n}$ and

$$
A=\left(\begin{array}{cc}
* & * \\
O & E
\end{array}\right) \text {, }
$$

where the asterisks represent suitable block matrices obtained to construct $A$ and

$$
E=T+F=\left(\begin{array}{cc}
T_{1} & O \\
O & T_{2}
\end{array}\right)+\left(\begin{array}{cc}
O & F_{1} \\
F_{2} & O
\end{array}\right),
$$

with

$$
\begin{aligned}
& T_{1}=\left(\begin{array}{ccccc}
q_{1}-\mu_{1} & 0 & \cdots & 0 & \\
\mu_{1} & q_{2}-\mu_{2} & \cdots & 0 & 0 \\
\vdots & \vdots & \ddots & \vdots & \vdots \\
0 & 0 & \cdots & \mu_{n-1} & q_{n}
\end{array}\right), \\
& T_{2}=\operatorname{diag}\left(s_{i}\right)_{i=1}^{n}, \\
& F_{j}=\left(\begin{array}{ccccc}
f_{1}^{j} 1 & 0 & \cdots & 0 & 0 \\
f_{21}^{j} & f_{22}^{j} & \cdots & 0 & 0 \\
\vdots & \vdots & \ddots & \vdots & \vdots \\
0 & 0 & \cdots & f_{n, n-1}^{j} & f_{n n}^{j}
\end{array}\right) \text {, }
\end{aligned}
$$

where $f_{i i}^{1}=\left(1-\epsilon_{i}\right) \alpha_{i} S_{i}^{f}, f_{i i}^{2}=\beta_{i}\left(1-\mu_{i}\right), i \in \Upsilon$ $\left(\epsilon_{n}=\mu_{n}=0\right)$ and $f_{i+1, i}^{1}=\epsilon_{i} \alpha_{i} S_{i}^{f}, f_{i+1, i}^{2}=\beta_{i} \mu_{i}$, $i=1, \ldots, n-1$.

To establish conditions on the population in order to ensure that the disease disappears, we consider the subsystem corresponding to the infected individuals and contaminant variables, $y(t+1)=E y(t)$. In what follows, we set $\mu_{n}=\epsilon_{n}=\sigma_{n}=0$.

Proposition 5. Consider the initial epidemic model described by the equations given in (6). If the population $P$ is distributed in compartments such that the DFE $P_{f}=\left(S^{f}, O, O\right) \in \mathbb{R}^{3 n}, S^{f}=\left(S_{1}^{f}, \ldots, S_{n}^{f}\right)$, satisfies

$$
S_{i}^{f}<K_{i}
$$

where

$$
K_{i}=\frac{\left(1-q_{i}+\mu_{i}\right)\left(1-s_{i}\right)}{\beta_{i}\left(1-\mu_{i}\right)\left(1-\epsilon_{i}\right) \alpha_{i}}, \quad i \in \Upsilon,
$$

then $x(t)$ is asymptotically stable to the DFE point $\left(S^{f}, O, O\right)$. Moreover, if $K_{i} \leq S_{i}^{f}$ for some $i, i \in \Upsilon$, then $x(t)$ is not asymptotically stable to this point.

Proof. Note that matrix $E$ is a nonnegative block one given as in (1). From the structure of $E$ we can apply the result given in Proposition 1 and obtain a similar system $z(t+1)=\tilde{E} z(t)$, where $\tilde{E}$ is the Frobenius normal form 
of $E$ and has the structure given in (1). In this case the blocks $\tilde{E}_{i}=\tilde{T}_{i}+\tilde{F}_{i}$ are given by

$$
\begin{aligned}
& \tilde{T}_{i}=\left(\begin{array}{cc}
q_{i}-\mu_{i} & 0 \\
0 & s_{i}
\end{array}\right), \\
& \tilde{F}_{i}=\left(\begin{array}{cc}
0 & \left(1-\epsilon_{i}\right) \alpha_{i} S_{i}^{f} \\
\beta_{i}\left(1-\mu_{i}\right) & 0
\end{array}\right), \quad i \in \Upsilon .
\end{aligned}
$$

If the system $y(t+1)=E y(t)$ is asymptotically stable to zero, then the initial system is asymptotically stable to the disease-free equilibrium point $\left(S^{f}, O, O\right)$ and the infection disappears. For this reason, we analyze the matrix $E$ given in (7).

The matrix $E=T+F$ is stable if and only if so are matrices $\tilde{E}_{i}=\tilde{T}_{i}+\tilde{F}_{i}$ for all $i \in \Upsilon$, or, equivalently, the spectral radius of matrix $\tilde{F}_{i}\left(I-\tilde{T}_{i}\right)^{-1}$ is less than 1 , for all $i \in \Upsilon$ (see, e.g., Berman and Plemmons, 1994; Li and Schneider, 2002). Finally, we can check that $\rho\left(\tilde{F}_{i}(I-\right.$ $\left.\left.\tilde{T}_{i}\right)^{-1}\right)<1$ if and only if the condition on $S_{i}^{f}$ given in (10) holds, $i \in \Upsilon$.

Remark 1. It is known that the basic reproductive number of the epidemiologic process, $R_{0}$, is a measure or an indicator of whether the disease will disappear ( $\mathrm{Li}$ and Schneider, 2002; Wijaya et al., 2017). If $R_{0}<1$, the disease tends to disappear around the disease-free equilibrium point and otherwise it remains. Note that the basic reproductive number of the epidemiologic process represented by the model (4) and defined by $R_{0}=$ $\rho\left(F(I-T)^{-1}\right)$ is less than 1 if and only if $R_{i}^{0}=\rho\left(\tilde{F}_{i}(I-\right.$ $\left.\left.\tilde{T}_{i}\right)^{-1}\right)<1$ for all $i \in \Upsilon$, with $\tilde{T}_{i}, \tilde{F}_{i}$ defined in $(8)$ and 9). Thus, from Proposition 5, the condition $R_{0}<1$ is satisfied if and only if the population in each compartment $S_{i}^{f}$ meets the condition (10).

Remark 2. Applying Proposition 5 to the components of $\left(S_{f}, O, O\right)$, with $S_{f}=\left(S_{1}^{f}, S_{2}^{f}, \ldots, S_{n}^{f}\right)$ given in Proposition 4, we obtain an upper bound over the total population $P$ in accordance with the expressions given in (5) and the conditions given in (10). In order to ensure that the contaminant population disappears, we need the population $S_{i}^{f}$ in each compartment to be lower than $K_{i}$, given in (10). In this case, the total population $P$ is less than

$$
\min \left\{\frac{K_{i} H}{H_{i-1, n} \Sigma_{i}}, i \in \Upsilon\right\} .
$$

\section{Seasonal actions to achieve the disappearance of the disease}

If $\mathcal{R}_{0} \geq 1$, some actions must be planned so that the basic reproduction number of the new model be less than 1. In many cases, the disease does not have an effective vaccine or there are other actions that are more profitable.
Typically, when there is an outbreak of Salmonella in a henhouse, it is not usual to vaccinate the hens for economic reasons. However, since the spread of infected individuals is caused by accumulation of the contaminant found in the environment, specifically in the floor of the henhouse, we can clean periodically. This leads to the mathematical representation of the process as an $N$-periodic system.

Firstly, we remark the following fact. The condition $\mathcal{R}_{0} \geq 1$, that is, $\rho(E) \geq 1$, implies that the population $P$ is greater than the bound given in 12 . Hence, there exists some $i, i \in \Upsilon$, such that $\rho\left(\tilde{E}_{i}\right) \geq 1$, that is, using Proposition 5, at the disease-free equilibrium point the population of the $i$-th compartment satisfies $K_{i} \leq S_{i}^{f}$.

On the other hand, each block $\tilde{E}_{i}$ given in 11) of the Frobenius normal form of matrix $E$ has the spectrum $\sigma\left(\tilde{E}_{i}=\left\{\lambda_{1,2}^{i}\right\}\right.$. We write $\rho\left(\tilde{E}_{i}\right)=\lambda_{1}^{i}$ and observe that the positive assumption of $\lambda_{2}^{i}$ is equivalent to

$$
S_{i}^{f}<L_{i}=\frac{\left(q_{i}-\mu_{i}\right) s_{i}}{\beta_{i}\left(1-\mu_{i}\right)\left(1-\epsilon_{i}\right) \alpha_{i}} .
$$

Thus, if, for some $i \in \Upsilon$, we have the conditions $\rho\left(\tilde{E}_{i}\right) \geq$ 1 and $\lambda_{2}^{i}>0$, then the parameters could satisfy the condition $K_{i}<S_{i}^{f}<L_{i}$.

Remark 3. Note that this condition is not too restrictive in the area of epidemiology since $K_{i}<L_{i}$ if and only if $q_{i}-\mu_{i}+s_{i}>1$, which means that the sum of the survival rates of infected individuals and the survival rate of the contaminant exceeds one. It is a realistic condition for many infectious diseases when an outbreak occurs, such as, e.g., Salmonella in a henhouse (see, e.g., Prevost et al., 2006).

To eliminate the infection, periodic cleaning of the environment is planned for each time $N$. From the initial condition $(S(0), I(0), 0), S(0)=\left(S_{i}(0)\right)_{i=1}^{n}$ and $I(0)=\left(I_{i}(0)\right)_{i=1}^{n}$, we clean the environment each $N$ steps leaving it without the contaminant. The new model is represented by (4), where $\alpha_{i}$ and $s_{i}$ are replaced by $\alpha_{i}(t)$ and $s_{i}(t)$, respectively, two $N$-periodic functions defined as

$$
\begin{gathered}
\alpha_{i}(t)= \begin{cases}0, & t=0, \\
\alpha_{i}, & t=1,2, \ldots, N-1,\end{cases} \\
s_{i}(t)= \begin{cases}0, & t=0, \\
s_{i}, & t=1,2, \ldots, N-1,\end{cases} \\
\alpha_{i}(t+N)=\alpha_{i}(t) \text { and } s_{i}(t+N)=s_{i}(t), i \in \Upsilon . \\
\text { Linearizing around the disease-free equil } \\
\text { point, we have that the subsystem correspond } \\
\text { the infected individuals and contaminant variable } \\
N \text {-periodic system } \\
\qquad \begin{array}{r}
E(t+1)=E(t) y(t), \\
E(t+N)=E(t), \quad t \geq 0,
\end{array}
\end{gathered}
$$

Linearizing around the disease-free equilibrium point, we have that the subsystem corresponding to the infected individuals and contaminant variables is an 
with $E(0)=E Q_{n}$ and $E(t)=E, t=1, \ldots, N-1$, where

$$
Q_{n}=\left(\begin{array}{cc}
J_{n} & O \\
O & O
\end{array}\right) \in \mathbb{R}^{2 n \times 2 n} .
$$

The solution of this system is given by means of the monodromy matrix defined as

$$
\Phi_{E}\left(k, k_{0}\right)= \begin{cases}\prod_{i=k_{0}}^{k-1} E\left(k_{0}+k-1-i\right), & k>k_{0} \\ J_{n}, & k=k_{0} .\end{cases}
$$

In this case, from an initial condition, $y_{0}=$ $(I(0), C(0)) \in \mathbb{R}^{2 n \times 2 n}$, the solution of the $N$-periodic system at time $t=k N+j, k \geq 0, j=1, \ldots, N-1$, is

$$
y(t)=\Phi_{E}(t, 0) y_{0}=E^{j} Q_{n}\left(\Phi_{E}(N, 0)\right)^{k} y_{0} .
$$

Using control theory tools developed for this kind of systems (see Bittanti, 1986), we know that the new $N$-periodic model will be asymptotically stable to zero if the monodromy matrix $\Phi_{E}(N, 0)$ is stable, that is, if $\rho\left(\Phi_{E}(N, 0)\right)<1$.

Note that if $N=1$, we have $C_{i}(t)=0, i \in \Upsilon$, for all $t$, and the system is asymptotically stable since $\rho\left(\Phi_{E}(1,0)\right)=\rho\left(E Q_{n}\right)=\max \left\{q_{i}-\mu_{i}, i \in \Upsilon\right\}<1$ and $0<\mu_{i}<q_{i}<1$. But we need to delay this cleaning action as much as possible. For this reason, we want to find the maximum number of steps at which the henhouse can remain without being cleaned, keeping the system asymptotically stable. That is, we want to obtain a threshold from which the system is not asymptotically stable and the disease does not tend to the disease-free equilibrium point. Then we look for the maximum period $N$ such that the $N$-periodic system (15) is asymptotically stable. Or, equivalently, we obtain the value of $N$ such that $\rho\left(\Phi_{E}(N, 0)\right)<1$ and $\rho\left(\Phi_{E}(N+1,0)\right)>1$, given $\Phi_{E}(N, 0)$. In this way, we establish the following result.

Theorem 2. Consider the $N$-periodic epidemiologic model given by (15), where matrix $E(t)=T(t)+F(t)$ is defined as in (7)-(9) with $\alpha_{i}$ and $s_{i}$ replaced by $\alpha_{i}(t)$ and $s_{i}(t)$ given in (13)-(14), $i \in \Upsilon$. Suppose that $S_{i}^{f}<L_{i}$ for all $i \in \Upsilon$, and for some $i, i \in \Upsilon, K_{i} \leq S_{i}^{f}$. Then there exists $N_{0} \in \mathbb{N}$ such that the $N$-period system (15) is asymptotically stable when the period $N<N_{0}$ and unstable when $N>N_{0}$.

Proof. Note that $\Phi_{E}(N, 0)=E^{N} Q_{n}$, with $E$ given by Eqns. (7)-(9). By constructing matrix $E=T+F$ from the parameters of the epidemiological process, each submatrix $\tilde{E}_{i}=\tilde{T}_{i}+\tilde{F}_{i}, i \in \Upsilon$, given by 111 , is a positive matrix with the entry $\tilde{e}_{11}^{i}=q_{i}-\mu_{i}<1$.

As there exists $i, i \in \Upsilon$, such that $K_{i} \leq S_{i}^{f}$, by Proposition 5, $\rho(E) \geq 1$. In addition, according to the comment at the beginning of this section, the condition $S_{i}^{f}<L_{i}$ yields $\lambda_{2}^{i}>0$, for all $i \in \Upsilon$. Thus, it is sufficient to apply Theorem 1 to assert that there exists $N_{0}$ such that $\rho\left(\Phi_{E}(N, 0)\right)<1$ if $N \leq N_{0}$ and $\rho\left(\Phi_{E}(N, 0)\right)>1$ when $N>N_{0}$.

\section{Illustrative example}

We analyze the evolution of an outbreak of Salmonella by the combination of three individual groups such that the transmission is via a free-living contaminant. This example is an academic one, but the values of the parameters considered are quite realistic and they are consistent with others used in studies that have analyzed this disease in a henhouse (see, e.g., Beaumont et al., 2012; Zongo et al., 2010). Consider a population $P=$ 208 , where the parameters involved in the model are

$$
\begin{gathered}
p_{i}=0.99, \quad q_{i}=0.8 \\
s_{i}=0.5, \quad i=1,2,3 \\
\alpha_{1}=0.00002, \quad \alpha_{2}=0.00001, \quad \alpha_{3}=0.00001 \\
\sigma_{1}=0.01, \quad \sigma_{2}=0.01 \\
\mu_{1}=0.001, \quad \mu_{2}=0.001 \\
\epsilon_{1}=0.0001, \quad \epsilon_{2}=0.0001
\end{gathered}
$$

in Step $^{-1}$ dimension, and the amount of excretion is

$$
\beta_{1}=10^{2} \mathrm{cfu}, \quad \beta_{2}=2.10^{2} \mathrm{cfu}, \quad \beta_{3}=3.10^{2} \mathrm{cfu},
$$

with 'cfu' standing for a colony forming unit.

The evolutions of the susceptible and infectious individuals and the contaminant are each compartment is given in Figs. 2 and 3, respectively.

The values of the disease-free equilibrium point $P_{e}$ are $S_{1}=104$ and $S_{2}=S_{3}=52$, and the coefficient matrix $E$ given in (7) of the linear system around this point has a spectral radius $\rho(E)=1.12921$. Then, the invariant system is unstable, but the conditions of Theorem $2, K_{i}<$ $S_{i}<L_{i}$ hold since $K_{1}=K_{2}=50.3053, K_{3}=33.33$, $L_{1}=L_{2}=199.97, L_{3}=133.33$. Here, we can ensure that there exists $N_{0} \in \mathbb{N}$ such that the $N$-periodic system (15) is asymptotically stable when the period $N<N_{0}$ and is not asymptotically stable when $N>N_{0}$.

In order to obtain the value of $N_{0}$, we need to construct the $N$-periodic matrix $E(t)$ as in (15) and analyze the spectral radius of the monodromy matrix.

As the conditions of the theorem are satisfied, we apply the algorithm constructed in Section 3 to the matrix $E$. Obtain that the submatrices

$$
\begin{gathered}
\tilde{E}_{1}=\left(\begin{array}{cc}
0.799 & 0.002 \\
99.9 & 0.5
\end{array}\right), \quad \tilde{E}_{2}=\left(\begin{array}{cc}
0.799 & 0519 \\
199.8 & 0.5
\end{array}\right), \\
\tilde{E}_{3}=\left(\begin{array}{cc}
0.8 & 0.00052 \\
300 & 0.5
\end{array}\right)
\end{gathered}
$$


have $\lambda_{1}^{1}=\rho\left(\tilde{E}_{1}\right)=1.129, \lambda_{1}^{2}=\rho\left(\tilde{E}_{2}\right)=1.0048$ and $\lambda_{1}^{3}=\rho\left(\tilde{E}_{3}\right)=1.0724$. Constructing the functions $f_{i}(z)$ of Step 5.2 of Algorithm 1 and solving $f_{i}(z)=0, i=$ $1,2,3$, we have

$$
N_{0}^{1}=3, \quad N_{0}^{2}=3, \quad N_{0}^{3}=5 .
$$

Then, $N_{0}=\min \left\{N_{0}^{i}, i=1,2,3\right\}=3$.

Indeed, checking the spectral radius of the monodromy matrix $\Phi_{E}(N, 0)$ of the $N$-periodic system according with its period, we have what follows: If $N=2, \rho\left(\Phi_{E}(2,0)\right)=0.8461$, if $N=3, \rho\left(\Phi_{E}(3,0)\right)=0.9459$ and if $N=4$,

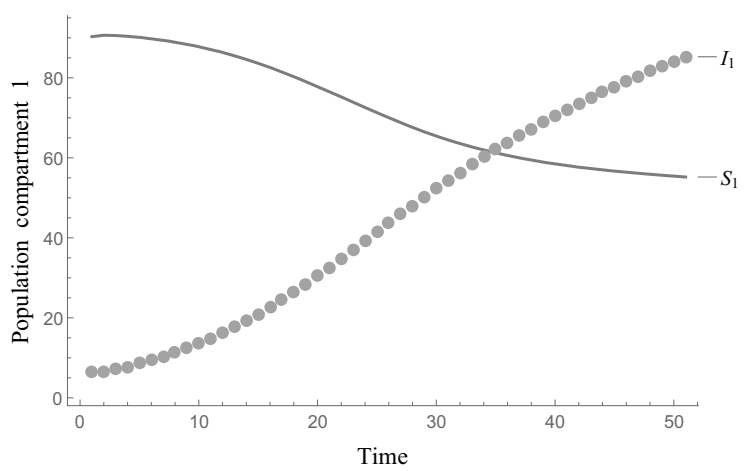

(a)

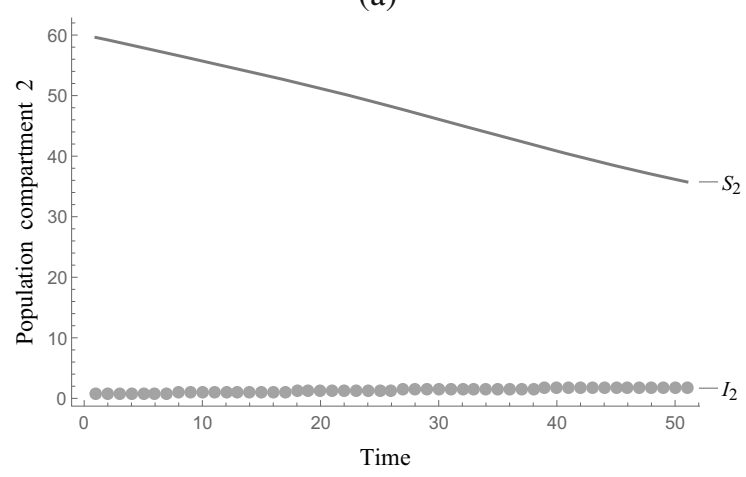

(b)

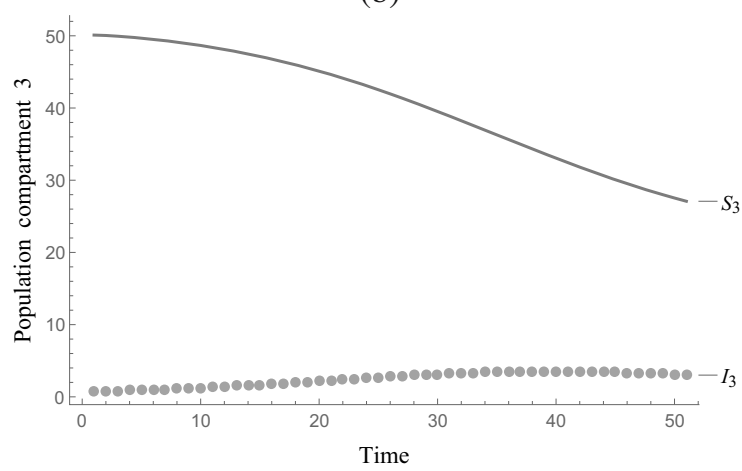

(c)

Fig. 2. Evolution of populations of susceptible and infected individuals: in compartment 1 (a), in compartment 2 (b), in compartment 3 (c).
$\rho\left(\Phi_{E}(4,0)\right)=1.066$.

Taking $N_{0}=3$, we have that the $N_{0}$-periodic system is asymptotically stable but the $\left(N_{0}+1\right)$-periodic system is not. In this way, the maximum number of steps the henhouse can be unclean, keeping the system asymptotically stable, is $N_{0}=3$. In Fig. 4 we can observe that the infected individuals in the first compartment tend to disappear when the enclosure is cleaned every three stages, eliminating the population of contaminant in the environment. However, if this seasonal cleaning is made every four steps, the disease cannot be completely eliminated.

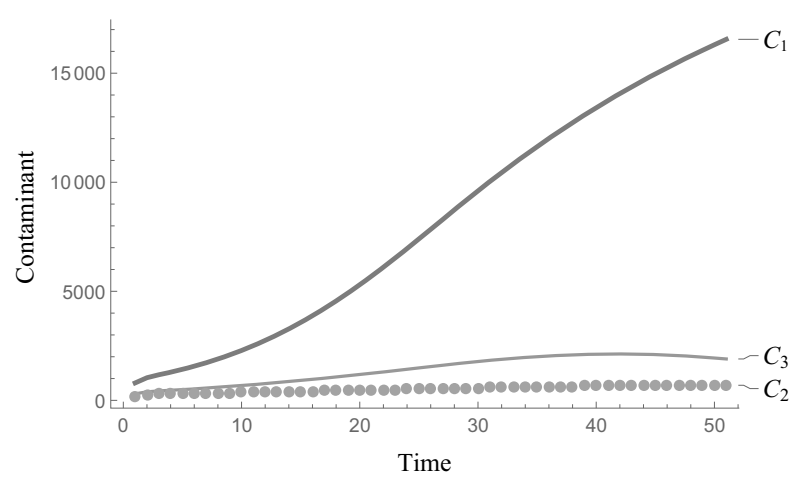

Fig. 3. Evolution of the contaminant in each compartment.

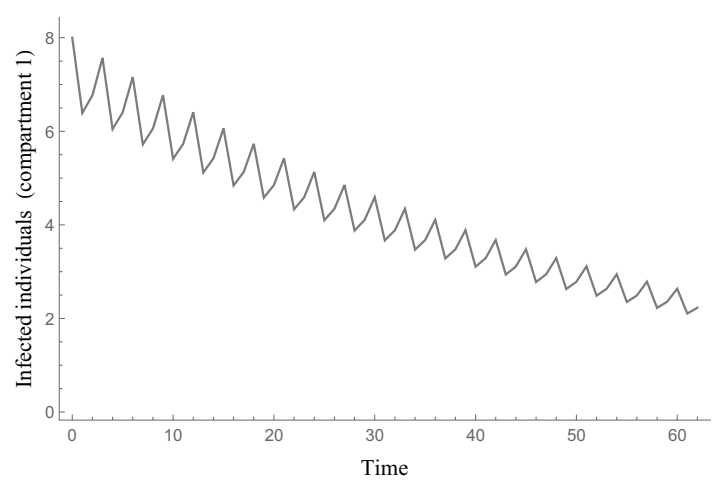

(a)

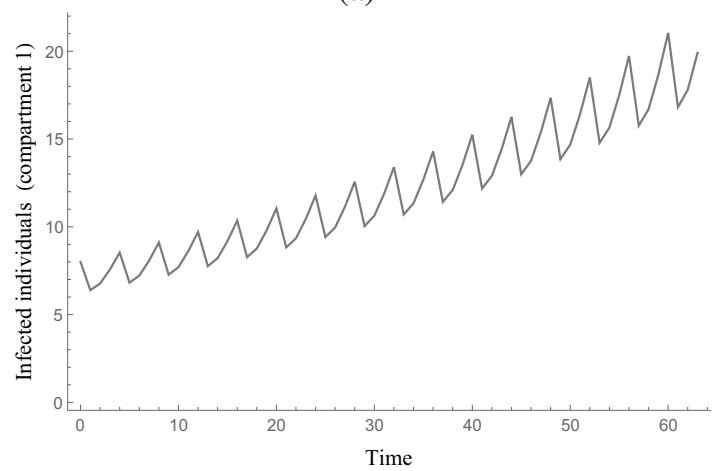

(b)

Fig. 4. Evolution of the population infected in compartment 1 when a seasonal cleanup strategy is set: periodic cleaning with $N=3$ (a), periodic cleaning with $N=4$ (b). 


\section{Conclusions}

In this paper, we presented a mathematical model to analyze the spread of Salmonella in an industrial henhouse. In this model, the individuals are in $n$ compartments and there is transference among the compartments. We studied the behavior of the model incorporating the contamination by the environment as an indirect mode of transmission. For that purpose, we used a linear approach which accurately reflects the behavior of the nonlinear model. Some properties of the matrices involved in the linear system were studied. These properties allowed us to obtain some conditions which determine whether or not the disease becomes extinct. Finally, we proposed a strategy to eliminate the possibility of infection. One of the main contributions of this work is the calculation of the maximal period of time such that this periodic cleaning of the environment allows us to prevent the spreading of the disease.

\section{Acknowledgment}

This work has been partially supported by the Spanish grant MTM2013-43678-P.

\section{References}

Ainseba, B.E., Benosman, C. and Maga, P. (2010). A model for ovine brucellosis incorporating direct and indirect transmission, Journal of Biological Dynamics 4(1): 2-11, DOI: $10.1080 / 17513750903171688$.

EFSA/ECLC (2014). The European Union summary report on trends and sources of zoonoses, zoonotic agents and food-borne outbreaks in 2012, EFSA Journal 12(2): 3547.

Beaumont, C., Burie, J., Ducrot, A. and Zongo, P. (2012). Propagation of Salmonella within an industrial hen house, SIAM Journal of Applied Mathematics 72(4): 1113-1148, DOI: $10.1137 / 110822967$.

Berman, A. and Plemmons, R. (1994). Nonnegative Matrices in the Mathematical Sciences, SIAM, Philadelphia, PA.

Bittanti, S. (1986). Deterministic and stochastic linear periodic systems, in S. Bittanti (Ed.), Time Series and Linear Systems, Lecture Notes in Control and Information Science, Vol. 86, Springer, Berlin, pp. 141-182.

Cantó, B., Coll, C. and Sánchez, E. (2013). Structured parametric epidemic model, International Journal of Computer Mathematics 91(2): 188-197, DOI: 10.1080/00207160.2013.800864.

Cantó, B., Coll, C. and Sánchez, E. (2014). A study on vaccination models for a seasonal epidemic process, $A p$ plied Mathematics and Computation 243: 152-160, DOI: 10.1016/j.amc.2015.05.104.

Ding, D., Ma, Q. and Ding, X. (2014). An unconditionally positive and global stability preserving NSFD scheme for an epidemic model with vaccination, International Journal of Applied Mathematics and Computer Science 24(3): 635-646, DOI: 10.2478/amcs-2014-0046.
Enatsu, Y., Nakata, Y. and Muroya, Y. (2012). Global stability for a discrete SIS epidemic model with immigration of infectives, Journal of Difference Equations and Applications 18(2): 1913-1924, DOI: 10.1080/10236198.2011.602973.

Joh, R., Wang, H., Weis, H. and Weitz, J. (2009). Dynamics of indirectly transmitted infectious diseases with immunological threshold, Bulletin of Mathematical Biology 71(4): 845-862.

Li, C. and Schneider, H. (2002). Applications of Perron-Frobenius theory to population dynamics, Journal Mathematical Biology 44(5): 450-462, DOI: 10.1007/s002850100132.

Li, X. and Wang, W. (2005). A discrete epidemic model with stage structure, Chaos Solitons \& Fractals 26(3): 947-958, DOI: 10.1016/j.chaos.2005.01.063.

Liao, S. and Yang, W. (2013). On the dynamics of a vaccination model with multiple transmission ways, International Journal of Applied Mathematics and Computer Science 23(4): 761-772, DOI: 10.2478/amcs-2013-0057.

Meyer, R. and Burrus, C. (1975). A unified analysis of multirate and periodically time-varying digital filters, IEEE Transactions on Circuits and Systems 22(3): 162-168.

Prevost, K., Beaumont, C. and Magal, P. (2006). Asymptotic behavior in a Salmonella infection model, Mathematical Modelling of Natural Phenomena 2(1): 1-22.

Rass, L. and Radcliffe, J. (2000). Global asymptotic convergence results for multitype models, International Journal of Applied Mathematics and Computer Science 10(1): 63-79.

van den Driessche, P. and Watmough, J. (2002). Reproduction numbers and sub-threshold endemic equilibria for compartmental models of disease transmission, Mathematical Biosciences 180(1): 29-48.

Wijaya, K.P., Sutimin, Soewono, E. and Götz, T. (2017). On the existence of a nontrivial equilibrium in relation to the basic reproductive number, International Journal of Applied Mathematics and Computer Science 27(3): 623-636, DOI: 10.1515/amcs-2017-0044.

Xiao, Y., Clancy, D., French, N. and Bowers, R. (2006). A semi-stochastic model for Salmonella infection in a multi-group herd, Mathematical Biosciences 200(2): 214-233.

Zongo, P., Viet, A., Magal, P. and Beaumont, C. (2010). A spatio-temporal model to describe the spread of Salmonella within a laying flock, Journal of Theoretical Biology 267(4): 595-604, DOI: 10.1016/j.jtbi.2010.09.030.

Begoña Cantó was born in Alcoi Alicante, Spain. She received the MEng degree in industrial organization engineering and the $\mathrm{PhD}$ degree in applied mathematics from Universitat Politècnica de Valencia, Spain. She is currently a professor in the Department of Applied Mathematics and a member of the Institute for Multidisciplinary Mathematics, Universitat Politècnica de Valencia. Her main research areas include periodic systems and linear control. She has published several papers in journals and international conferences and participated in several Spanish projects related to these topics. She is also interested in the reproduction number and stability of biological processes. 
Carmen Coll obtained her $\mathrm{PhD}$ degree at the Department of Applied Mathematics of the University of Valencia, Spain. Presently she is a full professor at the Engineering School of Universitat Politècnica de Valencia, Spain. She is a member of the Department of Mathematics and the Institute for Multidisciplinary Mathematics of that university. She has participated in the development of several research projects. She has published a number of articles in international journals and leading conferences. Her fields of interest include the area of applied mathematics, systems theory, especially singular multidimensional systems and positive multidimensional systems, mathematical modelling and identification. Her research is currently focused on the applications of mathematical modelling in medicine and biomedical engineering.
Elena Sánchez was born in Valencia, Spain. She received her PhD degree in applied mathematics from Universitat de València, Spain. Currently she is a professor at the Department of Applied Mathematics and a member of the Institute for Multidisciplinary Mathematics, Universitat Politècnica de València. Her main areas of research are matrix analysis and control systems theory. She works mainly on linear systems, as well as systems with periodic coefficients and nonnegative systems with applications to engineering, chemistry and biology. She has published several papers in journals and international conferences and participated in several Spanish projects related to these topics.

Received: 27 February 2017

Revised: 6 July 2017

Re-revised: 13 September 2017

Accepted: 25 September 2017 